\title{
High resolution spectral analysis of mixtures of complex exponentials modulated by polynomials
}

\author{
Roland Badeau, Member, IEEE, Bertrand David, and Gaël Richard, Member, IEEE
}

\begin{abstract}
High resolution methods such as the ESPRIT algorithm are of major interest for estimating discrete spectra, since they overcome the resolution limit of the Fourier transform and provide very accurate estimates of the signal parameters. In signal processing literature, most contributions focus on the estimation of exponentially modulated sinusoids in a noisy signal. In this paper, we introduce a more general class of signals, involving both amplitude and frequency modulations. We show that this Polynomial Amplitude Complex Exponentials (PACE) model is the most general model tractable by high resolution methods. We develop a generalized ESPRIT algorithm for estimating the signal parameters, and we show that this model can be characterized by means of a geometrical criterion.
\end{abstract}

Index Terms - high resolution, rotational invariance property, ESPRIT, polynomial modulation, multiple eigenvalues

\section{INTRODUCTION}

$\mathbf{T}$ He foundation of high resolution methods dates back from the work by Prony [1] published in 1795, which aims at estimating a sum of exponentials via linear prediction techniques. More recently, this approach was further investigated by Pisarenko [2] for estimating sinusoids in noise. On the other hand, modern high resolution methods rely on subspacebased signal analysis. This is the case of the MUltiple SIgnal Classification (MUSIC) algorithm [3] and its variant rootMUSIC [4], the Toeplitz Approximation Method (TAM) [5], the Estimation of Signal Parameters via Rotational Invariance Techniques (LS-ESPRIT) [6] and its variants TLS-ESPRIT [7] and PRO-ESPRIT [8]. In fact, all these estimation methods are also suitable for the more general Exponential Sinusoidal Model (ESM), which was successfully applied in the field of audio signal processing for example [9]-[11]. Additionally, specific estimation techniques were designed for the ESM, such as the Minimum-Norm (KT) method [12], the Matrix Pencil method [13] and the modified KT (MKT) method [14]. A survey of subspace-based signal analysis can be found in [15]. A different approach for estimating the parameters of the ESM is based on matching pursuit algorithms [16].

In signal processing literature, the ESM is generally considered as the general model tractable by high resolution methods. However, it can be shown that this model is restricted to signals which only contain single poles. Conversely, the more

Roland Badeau, Bertrand David and Gaël Richard are with the Department of Signal and Image Processing, École Nationale Supérieure des Télécommunications (ENST), Paris, France. E-mail: [roland.badeau, bertrand.david, gael.richard]@enst.fr.

(c) 2006 IEEE. Personal use of this material is permitted. However, permission to reprint/republish this material for advertising or promotional purposes or for creating new collective works for resale or redistribution to servers or lists, or to reuse any copyrighted component of this work in other works must be obtained from the IEEE. general Polynomial Amplitude Complex Exponentials (PACE) model proposed in this paper encompasses the multiple poles case. It describes a more general class of signals, involving both amplitude and frequency modulations, and leads to an alternative interpretation of the frequency estimates obtained by means of high resolution methods. A physical example of the PACE model is the critically damped simple harmonic motion of the spring / mass system, which involves a double pole. Below, a complete estimation scheme is proposed, based either on linear prediction or on the ESPRIT algorithm.

The paper is organized as follows. In section II, the solution to general homogeneous linear recursions is discussed, and a full parameterization is proposed. Then, it is demonstrated in section III that a Hankel data matrix containing successive samples of the signal is rank deficient and that its range space, known as the signal subspace, is spanned by a so-called Pascal-Vandermonde matrix. In section IV, the generalized ESPRIT method for estimating the PACE signal model is presented, and its performance regarding the estimation of amplitude and frequency modulated sinusoids is illustrated in section VI. Finally, the main conclusions of this paper are summarized in section VII, and some theoretical results are presented in the appendix.

\section{Discrete SIGNAL MODEL}

\section{A. Homogeneous linear recursions}

High resolution methods are historically linked to linear prediction techniques [1], [2], [12]. Indeed, all of them rely on the following discrete signal model:

$$
s(t)=\sum_{k=0}^{K-1} \alpha_{k} z_{k}^{t}
$$

where $K \in \mathbb{N}^{*}, \forall k \in\{0 \ldots K-1\} \alpha_{k} \in \mathbb{C}$, and all the poles $z_{k} \in \mathbb{C}^{*}$ are distinct. It is well known that such a signal satisfies an homogeneous linear recursion of the form

$$
s(t)+p_{1} s(t-1)+\ldots+p_{K} s(t-K)=0
$$

where $p_{1}, \ldots, p_{K}$, are the coefficients of the polynomial

$$
P[z]=\prod_{k=0}^{K-1}\left(z-z_{k}\right)
$$

written in the form $P[z]=\sum_{k=0}^{K} p_{(K-k)} z^{k}$, where $p_{0}=1$ and $p_{K} \neq 0$. Based on this observation, the estimation methods proposed in [1], [2], [12] consist in estimating the prediction polynomial $P[z]$ from the samples of the signal, whose roots form the estimated poles. 
If the signal is modeled as a sum of real or complex sinusoids, the poles are supposed to belong to the unit circle [1], [2]. Thus each pole $z_{k}$ can be written in the form $z_{k}=e^{i 2 \pi f_{k}}$ where $f_{k} \in \mathbb{R}$ is the frequency of the $k^{\text {th }}$ sinusoid. More generally, if the signal is modeled as a sum of exponentially modulated sinusoids (ESM), the poles can be anywhere in the complex plane except zero [12]. In this case, each pole $z_{k}$ can be written in the form $z_{k}=e^{\delta_{k}} e^{i 2 \pi f_{k}}$ where $\delta_{k} \in \mathbb{R}$ is the damping factor of the $k^{\text {th }}$ sinusoid. In particular, poles with the same polar angle and different radii are associated to the same frequency.

Nevertheless, the ESM does not correspond to the general solution of homogeneous linear recursions, since in the general case a prediction polynomial can have multiple roots. To handle this case, equation (2) must be replaced by

$$
P[z]=\prod_{k=0}^{K-1}\left(z-z_{k}\right)^{M_{k}}
$$

where $\forall k \in\{0 \ldots K-1\}, M_{k} \in \mathbb{N}^{*}$ can be greater than 1 , so that the degree of $P[z]$ is $r=\sum_{k=0}^{K-1} M_{k} \geq K$. Thus the prediction polynomial can be written in the form $P[z]=$ $\sum_{k=0}^{r} p_{(r-k)} z^{k}$, where $p_{0}=1$ and $p_{r} \neq 0$. The general solution to the corresponding linear recursion

$$
s(t)+p_{1} s(t-1)+\ldots+p_{r} s(t-r)=0
$$

is obtained by turning equation (1) into

$$
s(t)=\sum_{k=0}^{K-1} \alpha_{k}[t] z_{k}^{t}
$$

where $\forall k \in\{0, \ldots, K-1\}, \alpha_{k}[t]$ is a complex polynomial of order less or equal to $M_{k}-1$ (see [17, pp. 33] for a proof). In this paper, the signal model in equation (4) is referred to as the Polynomial Amplitude Complex Exponentials (PACE) model. In particular, this model can associate several single poles to a single frequency (as for the ESM), as well as multiple poles (contrary to the ESM).

\section{B. Full parameterization of the signal model}

The signal model in equation (4) is not yet complete, since a full parameterization would additionally require the choice of a polynomial basis over which $\alpha_{k}[t]$ could be projected. Below, we focus on a particular polynomial basis which satisfies a simple linear recursion.

Definition II.1 (Falling factorial). For all $m \in \mathbb{Z}$, the falling factorial of order $m$ is the polynomial ${ }^{1}$

$$
F_{m}[X]= \begin{cases}0 & \text { if } m<0 \\ 1 & \text { if } m=0 \\ \frac{1}{m !} \prod_{m^{\prime}=0}^{m-1}\left(X-m^{\prime}\right) & \text { if } m>0\end{cases}
$$

\footnotetext{
${ }^{1}$ Note that this definition does not exactly match the classical definition of the falling factorial [18], [19], from which the multiplicative factor $\frac{1}{m !}$ is missing.
}

The family $\left\{F_{m}[X]\right\}_{m \geq 0}$ is a basis of $\mathbb{C}[X]$ since the degree of $F_{m}[X]$ is $m$ for all $m \geq 0$. In addition, these polynomials satisfy for all $m \in \mathbb{Z}$ the linear recursion

$$
F_{m}[t+1]=F_{m}[t]+F_{m-1}[t] \quad \forall t \in \mathbb{Z} .
$$

The polynomials $\alpha_{k}$ of order $M_{k}-1$ can be decomposed into the basis $\left\{F_{m}[X]\right\}_{m \geq 0}: \forall k \in\{0 \ldots K-1\}$,

$$
\alpha_{k}[X]=\sum_{m=0}^{M_{k}-1} \alpha_{(k, m)}^{\prime} F_{m}[X]
$$

where $\forall m \in\left\{0 \ldots M_{k}-1\right\}, \alpha_{(k, m)}^{\prime} \in \mathbb{C}$, so that equation (4) can be rewritten in the form ${ }^{2}$

$$
s(t)=\sum_{k=0}^{K-1} \sum_{m=0}^{M_{k}-1} \alpha_{(k, m)} F_{m}[t] z_{k}^{t-m}
$$

where $\forall k \in\{0 \ldots K-1\}, \forall m \in\left\{0, M_{k}-1\right\}, \alpha_{(k, m)}=$ $\alpha_{k, m}^{\prime} z_{k}^{m}$ is a complex amplitude.

This signal model can be extended by introducing an additive noise. More precisely, the observed signal $x(t)$ can be modeled as the sum of the deterministic signal $s(t)$ defined in equation (6), plus an additive white noise $w(t)$ of variance $\sigma^{2}: x(t)=s(t)+w(t)$.

Therefore, the parameters of the complete model are:

- the order $K$ and the multiplicities $\left\{M_{k}\right\}_{k \in\{0 \ldots K-1\}}$,

- the $K$ complex poles $z_{k}$,

- the $r$ complex amplitudes $\alpha_{(k, m)}$,

- the variance $\sigma^{2}$.

High resolution methods based on linear prediction, such as [1], [2], [12], can be used directly to estimate the parameters $K, M_{k}$ and $z_{k}$, which are completely characterized by the prediction polynomial. However, in a noisy context, the estimated prediction polynomial does not have multiple roots. This problem will be discussed in section V-A.

Remark. The modeling order for both the ESM and the PACE model is the order of the prediction polynomial $P[z]$. Thus it would be interesting to compare the numbers of parameters involved by the two models for a same modeling order $r$. Indeed, the PACE model is interesting for coding applications because all the poles $z_{k}$ of multiplicity $M_{k}>1$ only need to be coded one time. However, the multiplicities $\left\{M_{k}\right\}_{k \in\left\{0 \ldots M_{k}-1\right\}}$ also have to be coded, which is not the case for the ESM, which only contains single poles. More precisely, the PACE model involves $K+1$ integers, plus $2 K+2 r+1$ real numbers. As a comparison, the ESM model involves 1 integer (the model order), plus $4 r+1$ real numbers. We can conclude that the PACE model involves less parameters than the ESM when $K \leq \frac{2}{3} r$. Besides, integers can be coded with less bits than real numbers, which suggests that the PACE model can also be interesting even if this inequality is not satisfied.

\section{MATRIX MODEL}

As opposed to linear prediction techniques, modern high resolution methods (e.g. [3], [6], [13]) rely on matrix analysis (more precisely on the particular structure of the data matrix).

\footnotetext{
${ }^{2}$ The intentional introduction of the time shift $t-m$ will be self-explanatory in the following developments.
} 


\section{A. Definition and range space of the data matrix}

The samples of a discrete signal $s(t)$ can be arranged into a Hankel data matrix with $n \in \mathbb{N}^{*}$ rows and $l \in \mathbb{N}^{*}$ columns:

$$
\boldsymbol{S}(t)=\left[\begin{array}{cccc}
s(t-l+1) & \cdots & s(t-1) & s(t) \\
s(t-l+2) & \cdots & s(t) & s(t+1) \\
\vdots & \cdots & \vdots & \vdots \\
s(t-l+n) & \cdots & s(t+n-2) & s(t+n-1)
\end{array}\right]
$$

The following theorem shows that if the matrix $\boldsymbol{S}(t)$ has a deficient rank $r<\min (n, l)$ then the observed signal satisfies the noiseless model (6) under a simple condition.

Theorem III.1 (Equivalence of the low-rank Hankel structure and the signal model). Let $n \geq 2, l \geq 2$, and $r$ an integer such that $r<n$ and $r<l$. Let $\boldsymbol{S}(t)$, be the matrix extracted from $\boldsymbol{S}(t)$ by deleting the last row. Similarly, let $\boldsymbol{S}(t)_{\uparrow}$ be the matrix extracted from $\boldsymbol{S}(t)$ by deleting the first row. The following statements are equivalent:

1) The matrix $\boldsymbol{S}(t)$ has rank $r$, and the extracted matrices $\boldsymbol{S}(t) \downarrow$ and $\boldsymbol{S}(t) \uparrow$ also have rank $r$.

2) The signal $s(t)$ can be written in the form (6) on the interval $[t-l+1 \ldots t+n-1]$, and $\forall k \in\{0 \ldots K-1\}$, $\alpha_{\left(k, M_{k}-1\right)} \neq 0$.

The proof of this theorem is quite complex and can be found in [20].

Below, we only assume that $s(t)$ can be written in the form (6) on the interval $[t-l+1 \ldots t+n-1]$, without supposing that $\forall k \in\{0 \ldots K-1\}, \alpha_{\left(k, M_{k}-1\right)} \neq 0$. In order to characterize the range space of $\boldsymbol{S}(t)$, we need to introduce the so-called generalized Pascal and Pascal-Vandermonde matrices. First, generalized Pascal matrices form a generalization of the well known lower triangular Pascal matrices ${ }^{3}$, whose definition can be found in [21].

Definition III.2 (Generalized Pascal matrices). Let $z \in \mathbb{C}$ and $M \in \mathbb{N}^{*}$. The generalized Pascal matrix denoted $C_{M}^{n}(z)$ is a $n \times M$ matrix whose coefficients are $C_{M}^{n}(z)_{(i, j)}=F_{j}[i] z^{i-j}$ for all $i \in\{0 \ldots n-1\}$ and $j \in\{0 \ldots M-1\}$.

Example III.3. If $M=3$ and $n=7$,

$$
\boldsymbol{C}_{3}^{7}(z)=\left[\begin{array}{lll}
1 & 0 & 0 \\
z & 1 & 0 \\
z^{2} & 2 z & 1 \\
z^{3} & 3 z^{2} & 3 z \\
z^{4} & 4 z^{3} & 6 z^{2} \\
z^{5} & 5 z^{4} & 10 z^{3} \\
z^{6} & 6 z^{5} & 15 z^{4}
\end{array}\right]
$$

A Pascal-Vandermonde matrix is formed by concatenating several generalized Pascal matrices. Thus the following definition generalizes the classical Vandermonde structure [22, pp. 29]. It can also be found in [23].

\footnotetext{
${ }^{3}$ A lower triangular Pascal matrix is a square generalized Pascal matrix for which $z=1$.

${ }^{4}$ If $z=0$, we define $C_{M}^{n}(0)_{(i, i)}=1 \forall i$, and $\forall i \neq j, C_{M}^{n}(0)_{(i, j)}=0$.
}

Definition III.4 (Pascal-Vandermonde matrices). Let $K \in \mathbb{N}^{*}$. For all $k \in\{0 \ldots K-1\}$, let $z_{k} \in \mathbb{C}$ and $M_{k} \in \mathbb{N}^{*}$. Let $r \triangleq \sum_{k=0}^{K-1} M_{k}$. The Pascal-Vandermonde matrix is the $n \times r$ matrix formed by concatenating the generalized Pascal matrices $C_{M_{k}}^{n}\left(z_{k}\right)$ :

$$
\boldsymbol{V}^{n}=\left[\boldsymbol{C}_{M_{0}}^{n}\left(z_{0}\right), \ldots, \boldsymbol{C}_{M_{K-1}}^{n}\left(z_{(K-1)}\right)\right] .
$$

Example III.5. If $K=4,\left\{M_{0}, M_{1}, M_{2}, M_{3}\right\}=\{1,3,1,2\}$, and $n=r=7$,

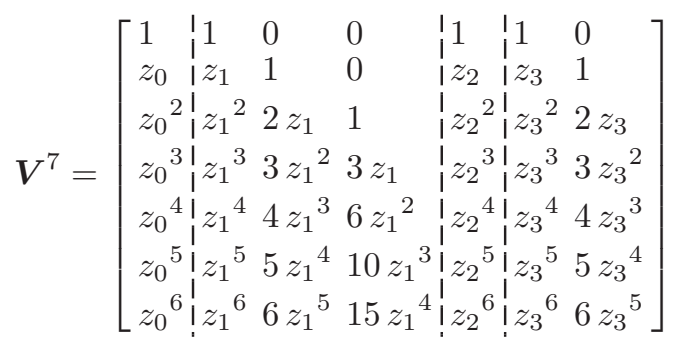

The following proposition generalizes a classical result about the determinant of Vandermonde matrices [22, pp. 29].

Proposition III.6 (Determinant and rank of Pascal-Vandermonde matrices). The determinant of the square PascalVandermonde matrix $\boldsymbol{V}^{r}$ is

$$
\prod_{\substack{k_{1}, k_{2}=0 \\ k_{1}<k_{2}}}^{K-1}\left(z_{k_{2}}-z_{k_{1}}\right)^{M_{k_{1}} M_{k_{2}}}
$$

As a result, the $n \times r$ Pascal-Vandermonde matrix $\boldsymbol{V}^{n}$ (with $n \geq r$ ) has rank $r$ if and only if the $K$ parameters $z_{0}, \ldots, z_{K-1}$ are distinct.

The proof of this proposition can be found in [20].

\section{B. Factorization of the data matrix}

Based on the above definitions, a factorization of the Hankel data matrix is proposed in this section ${ }^{5}$. Proposition III.7 is a generalization of the result presented in [13] to the multiple poles case.

Proposition III.7 (Factorization of the data matrix). A $n \times l$ Hankel matrix $\boldsymbol{S}(t)$ of the form (7) where $s(t)$ is the signal defined in equation (6) can be factorized in the form

$$
\boldsymbol{S}(t)=\boldsymbol{V}^{n} \boldsymbol{D}(t) \boldsymbol{V}^{l^{T}}
$$

where $\boldsymbol{D}(t)$ is the $r \times r$ block-diagonal matrix

$$
\boldsymbol{D}(t)=\left[\begin{array}{cccc}
\boldsymbol{H}_{0}(t) & 0 & \cdots & 0 \\
0 & \boldsymbol{H}_{1}(t) & \ddots & \vdots \\
\vdots & \ddots & \ddots & 0 \\
0 & \cdots & 0 & \boldsymbol{H}_{(K-1)}(t)
\end{array}\right]
$$

${ }^{5}$ Such a factorization was already established by Vandevoorde and Boley in [23], [24]. However, the developments presented here rely on different concepts. Moreover, they lead to an explicit formulation of the block-diagonal factor $\boldsymbol{D}(t)$ (see proposition III.7). 
whose $k^{\text {th }}$ block $\boldsymbol{H}_{k}(t)$ is the $M_{k} \times M_{k}$ upper anti-triangular Hankel matrix

$$
\boldsymbol{H}_{k}(t)=\left[\begin{array}{cccc}
\beta_{(k, 0)}(t) & \beta_{(k, 1)}(t) & \cdots & \beta_{\left(k, M_{k}-1\right)}(t) \\
\beta_{(k, 1)}(t) & \cdot \cdot & \cdot \cdot & 0 \\
\vdots & . \cdot & . \cdot & \vdots \\
\beta_{\left(k, M_{k}-1\right)}(t) & 0 & \cdots & 0
\end{array}\right]
$$

whose coefficients are, for all $k \in\{0 \ldots K-1\}$ and $m^{\prime} \in$ $\left\{0 \ldots M_{k}-1\right\}$,

$$
\beta_{k, m^{\prime}}(t)=\sum_{m=m^{\prime}}^{M_{k}-1} \alpha_{(k, m)} F_{m-m^{\prime}}[t-l+1] z_{k}^{t-l+1-\left(m-m^{\prime}\right)}
$$

The proof of proposition III.7 is presented in appendix A. The following corollary is the foundation of the estimation technique presented in section IV.

Corollary III.8 (Rank of the data matrix). A $n \times l$ Hankel matrix $\boldsymbol{S}(t)$ of the form (7), where $s(t)$ is the signal defined in equation (6), has rank $r$ if and only if

$$
\forall k \in\{0 \ldots K-1\}, \alpha_{\left(k, M_{k}-1\right)} \neq 0 .
$$

The proof of corollary III.8 can be found in appendix A. In section IV, it is always supposed that condition (11) is satisfied. This condition means that $\forall k \in\{0, \ldots, K-1\}$, the polynomial $\alpha_{k}[t]$ in equation (4) is of order $M_{k}-1$.

\section{ESTIMATION OF THE MODEL PARAMETERS}

Below, the generalized ESPRIT algorithm for estimating the poles independently from the complex amplitudes is presented. Then methods for estimating the model order $r$ and the complex amplitudes are briefly reviewed in section IV-D.

\section{A. Low-rank structure of the correlation matrix}

Subspace-based methods rely on the particular structure of the correlation matrix of the signal $s(t)$, which is defined as follows :

$$
\boldsymbol{R}_{s s}(t)=\frac{1}{l} \boldsymbol{S}(t) \boldsymbol{S}(t)^{H} .
$$

Substituting equation (8) into equation (12) yields the following factorization of $\boldsymbol{R}_{s s}(t)$ :

$$
\boldsymbol{R}_{s s}(t)=\boldsymbol{V}^{n} \boldsymbol{P}(t) \boldsymbol{V}^{n H}
$$

where $\boldsymbol{P}(t)$ is the $r \times r$ time-varying positive definite matrix

$$
\boldsymbol{P}(t)=\frac{1}{l} \boldsymbol{D}(t) \boldsymbol{V}^{l^{T}} \boldsymbol{V}^{l^{*}} \boldsymbol{D}(t)^{H} .
$$

In particular, equation (13) shows that the $n \times r$ matrix $\boldsymbol{V}^{n}$ spans the $r$-dimensional range space of $\boldsymbol{R}_{s s}(t)$, which is called signal subspace in the literature. Since $\boldsymbol{R}_{s s}(t)$ is a positive semidefinite matrix, it is diagonalizable in an orthonormal basis, and its eigenvalues are non-negative. Moreover, since $\boldsymbol{R}_{s s}(t)$ has rank $r<n, r$ of its eigenvalues are positive, whereas the $n-r$ other ones are zero.

Similarly to equation (12), define the $n \times n$ correlation matrix of the noisy signal $x(t)$ :

$$
\widehat{\boldsymbol{R}}_{x x}(t)=\frac{1}{l} \boldsymbol{X}(t) \boldsymbol{X}(t)^{H}
$$

where the data matrix $\boldsymbol{X}(t)$ is defined from the noisy signal $x(t)$ in the same way as $\boldsymbol{S}(t)$ in equation (7). Since the additive noise $w(t)$ is white and centered, of variance $\sigma^{2}$, the expectation matrix $\boldsymbol{R}_{x x}(t)=\mathbb{E}\left[\widehat{\boldsymbol{R}}_{x x}(t)\right]$ satisfies

$$
\boldsymbol{R}_{x x}(t)=\boldsymbol{R}_{s s}(t)+\sigma^{2} \boldsymbol{I}_{n} .
$$

This last equation shows that all the eigenvectors of $\boldsymbol{R}_{s s}(t)$ are eigenvectors of $\boldsymbol{R}_{x x}(t)$, and that the eigenvalues of $\boldsymbol{R}_{x x}(t)$ are equal to those of $\boldsymbol{R}_{s s}(t)$ plus $\sigma^{2}$. Therefore, the range space $\boldsymbol{R}_{x x}(t)$ (called signal subspace) is also the $r$-dimensional principal subspace of $\boldsymbol{R}_{x x}(t)$, i.e. the eigensubspace of $\boldsymbol{R}_{x x}(t)$ associated to the $r$ eigenvalues of magnitude strictly higher than $\sigma^{2}$ (the $n-r$ other ones being equal to $\sigma^{2}$ ).

\section{B. Rotational Invariance Property}

The ESPRIT estimation method relies on a particular property of Vandermonde matrices known as the rotational invariance [6], which reflects the invariance of the signal subspace to time shifts. Theorem IV.1 generalizes this property to Pascal-Vandermonde matrices.

Theorem IV.1 (Rotational Invariance Property of Pascal-Vandermonde matrices). Suppose that $n \geq 2$. Let $\boldsymbol{V}_{\downarrow}^{n}$ be the matrix extracted from $\boldsymbol{V}^{n}$ by deleting the last row. Similarly, let $\boldsymbol{V}_{\uparrow}^{n}$ be the matrix extracted from $\boldsymbol{V}^{n}$ by deleting the first row. Then $\boldsymbol{V}_{\downarrow}^{n}$ and $\boldsymbol{V}_{\uparrow}^{n}$ span the same subspace, and

$$
\boldsymbol{V}_{\uparrow}^{n}=\boldsymbol{V}_{\downarrow}^{n} \boldsymbol{J}
$$

where $\boldsymbol{J}$ is the $r \times r$ block-diagonal matrix

$$
\boldsymbol{J}=\left[\begin{array}{cccc}
\boldsymbol{J}_{M_{0}}\left(z_{0}\right) & 0 & \cdots & 0 \\
0 & \boldsymbol{J}_{M_{1}}\left(z_{1}\right) & \ddots & \vdots \\
\vdots & \ddots & \ddots & 0 \\
0 & \cdots & 0 & \boldsymbol{J}_{M_{(K-1)}\left(z_{(K-1)}\right)}
\end{array}\right]
$$

whose $k^{\text {th }}$ block $\boldsymbol{J}_{M_{k}}\left(z_{k}\right)$ is the $M_{k} \times M_{k}$ Jordan block

$$
\boldsymbol{J}_{M_{k}}\left(z_{k}\right)=\left[\begin{array}{ccccc}
z_{k} & 1 & 0 & \ldots & 0 \\
0 & z_{k} & 1 & \ddots & \vdots \\
0 & 0 & z_{k} & \ddots & 0 \\
\vdots & \ddots & \ddots & \ddots & 1 \\
0 & \ldots & 0 & 0 & z_{k}
\end{array}\right] \text {. }
$$

Theorem IV.1 is a corollary of lemma B.1, presented in appendix B. The interesting fact in theorem IV.1 is that equation (15) involves a Jordan matrix $\boldsymbol{J}$, which characterizes the poles $z_{k}$ and their multiplicity $M_{k}$. As shown below, the generalized ESPRIT algorithm consists in computing $\boldsymbol{J}$ as a by-product of the Jordan canonical decomposition ${ }^{6}$ of a socalled spectral matrix.

\footnotetext{
${ }^{6}$ See [22, pp. 121-142] for a definition of the Jordan canonical decomposition.
} 


\section{The generalized ESPRIT method}

In practice, the Pascal-Vandermonde matrix $\boldsymbol{V}^{n}$ is unknown, but a $n \times r$ matrix $\boldsymbol{W}(t)$ spanning the signal subspace can be estimated by computing the eigenvalue decomposition of $\widehat{\boldsymbol{R}}_{x x}(t)$, or the singular value decomposition of $\boldsymbol{X}(t)$, or by means of subspace tracking techniques [25]-[27]. Since $\boldsymbol{W}(t)$ and $\boldsymbol{V}^{n}$ span the same subspace, there is a non-singular matrix $\boldsymbol{G}(t)$ of dimension $r \times r$ such that

$$
\boldsymbol{V}^{n}=\boldsymbol{W}(t) \boldsymbol{G}(t) .
$$

Substituting equation (17) into equation (15) shows that $\boldsymbol{W}(t)$ satisfies an equation similar to equation (15):

$$
\boldsymbol{W}(t)_{\uparrow}=\boldsymbol{W}(t)_{\downarrow} \boldsymbol{\Phi}(t)
$$

where $\boldsymbol{\Phi}(t)$, herein called the spectral matrix, is defined by its Jordan canonical decomposition:

$$
\boldsymbol{\Phi}(t) \triangleq \boldsymbol{G}(t) \boldsymbol{J} \boldsymbol{G}(t)^{-1} .
$$

Finally, the generalized ESPRIT algorithm consists of the following steps:

- estimate a basis $\widehat{\boldsymbol{W}}(t)$ of the signal subspace,

- compute an estimator $\widehat{\boldsymbol{\Phi}}(t)$ of the spectral matrix, using a $\mathrm{LS}^{7}$ or $\mathrm{TLS}^{8}$ technique.

- compute the eigenvalues of $\widehat{\boldsymbol{\Phi}}(t)$ from which the estimated poles and their multiplicities can be extracted.

Note that in a noisy context, the estimated spectral matrix does not have multiple eigenvalues in practice, and the generalized ESPRIT algorithm cannot be applied as it is. This problem will be discussed in section V-B.

\section{Estimation of the other parameters}

We now focus on the estimation of the model order $r$ and the complex amplitudes $\alpha_{k, m}$.

1) Estimation of the modeling order: In the above discussion, the model order is supposed to be known, which is not the case in practice. Many methods were proposed in the literature for estimating the number of sinusoids in white noise. The most classical ones are the maximum likelihood method [28] and the information theoretic criteria, among which the Akaike Information Criterion (AIC) and the Maximum Description length (MDL) [29], and their generalization known as the Efficient Detection Criterion [30]. In [31], [32], we proposed a conceptually different approach which minimizes the frequency estimation bias. This method can be applied directly for estimating the order of the PACE signal model.

2) Estimation of the complex amplitudes: The Least Squares (LS) and Weighted Least Squares (WLS) are very classical methods for estimating the amplitudes of sinusoids of known frequencies corrupted by noise. A good survey of such techniques was proposed in [33]. Again, these methods can be adapted in a straight manner for estimating the complex amplitudes of the PACE signal model.

\footnotetext{
${ }^{7}$ The classical LS-ESPRIT method [6] computes $\widehat{\boldsymbol{\Phi}}(t)=\widehat{\boldsymbol{W}}(t) \dagger \widehat{\boldsymbol{W}}(t) \uparrow$ (where the symbol $\dagger$ denotes the Moore-Penrose pseudo-inverse).

${ }^{8}$ The TLS-ESPRIT algorithm estimates $\widehat{\boldsymbol{\Phi}}(t)$ as the solution of a Total Least Squares (TLS) minimization problem [7].
}

\section{Perturbation ANAlysis}

In this section, we illustrate how sensitive single and multiple poles are to perturbations. Linear prediction-based high resolution methods are analyzed in section $\mathrm{V}-\mathrm{A}$, and the generalized ESPRIT method is analyzed in section V-B.

\section{A. Perturbation of the prediction polynomial}

As mentioned in section II-B, high resolution methods based on linear prediction, such as [1], [2], [12], estimate the parameters $K, M_{k}$ and $z_{k}$ by computing the roots of the prediction polynomial $P[t]$.

In practice, contrary to $P[t]$, the estimated prediction polynomial does not have multiple roots. Indeed, the additive noise $w(t)$ perturbs the estimated coefficients, so that each multiple root of $P[t]$ is scattered into several single roots. The estimated prediction polynomial is denoted $P_{\varepsilon}[z]=P[z]+\varepsilon \Delta P[z]$, where $\Delta P[z]$ is a polynomial of order lower than $r$, and $\varepsilon$ is supposed to be small. In practice, the deviation $\varepsilon \Delta P[z]$ depends both on the noise $w(t)$ and on the particular method used to estimate the prediction polynomial, such as [1], [2], [12].

Proposition V.1. Let $z_{k}$ be a root of multiplicity $M_{k} \in \mathbb{N}^{*}$ of the $r^{\text {th }}$ order polynomial $P[z]$. For all $\varepsilon>0$, let $P_{\varepsilon}[z]=$ $P[z]+\varepsilon \Delta P[z]$, where $\Delta P[z]$ is a polynomial of order lower than $r$. Suppose that $\Delta P\left[z_{k}\right] \neq 0$. Then there exists a positive $\varepsilon_{0}$ such that for all $\varepsilon<\varepsilon_{0}$ there are exactly $M_{k}$ roots of $P_{\varepsilon}[z]$, denoted $\left\{z_{(k, m)}(\varepsilon)\right\}_{m \in\left\{0 \ldots M_{k}-1\right\}}$, which admit the first-order fractional expansion

$$
z_{(k, m)}(\varepsilon)=z_{k}+\varepsilon^{\frac{1}{M_{k}}} \Delta z_{k} e^{i 2 \pi \frac{m}{M_{k}}}+O\left(\varepsilon^{\frac{2}{M_{k}}}\right)
$$

where $\Delta z_{k}$ is an arbitrary $M_{k}^{\text {th }}$ root of the complex number

$$
\left(\Delta z_{k}\right)^{M_{k}}=-\frac{\Delta P\left[z_{k}\right]}{\frac{1}{M_{k} !} P^{\left(M_{k}\right)}\left[z_{k}\right]} .
$$

This proposition is related to a classical result about algebraic functions [34, pp. 64-66]. Note that in equation (19) the first order perturbation of $z_{k}$ is homogeneous and isotropic, so that the $M_{k}$ perturbed roots form the vertices of a $M_{k}{ }^{\text {th }}$ order regular polygon in the complex plane. This may be a way of discriminating between several perturbed single poles and a single perturbed multiple pole (when $M_{k} \geq 3$ ). Multiple poles appear to be more sensitive to perturbations than single poles, since the first order term in equation (19) is $\varepsilon^{\frac{1}{M_{k}}}$. In fact, this apparent sensitivity can be circumvented by taking the multiplicity structure of the polynomial $P[z]$ in equation (3) into account [35].

\section{B. Perturbation of the spectral matrix}

In the case of the generalized ESPRIT method, the poles are obtained by computing the eigenvalues of the spectral matrix $\boldsymbol{\Phi}(t)$. In practice, contrary to $\boldsymbol{\Phi}(t)$, the estimated spectral matrix does not have multiple eigenvalues. As mentioned in section $\mathrm{V}-\mathrm{A}$ in the case of linear prediction techniques, the additive noise $w(t)$ perturbs the estimation, so that each multiple eigenvalue of $\boldsymbol{\Phi}(t)$ is scattered into several single eigenvalues. 
The estimated spectral matrix is denoted $\boldsymbol{\Phi}_{\varepsilon}(t)=\boldsymbol{\Phi}(t)+$ $\varepsilon \Delta \boldsymbol{\Phi}(t)$, where $\Delta \boldsymbol{\Phi}(t)$ is a $r \times r$ matrix, and $\varepsilon$ is supposed to be small. In practice, the deviation $\varepsilon \Delta \Phi(t)$ depends both on the noise $w(t)$ and on the particular method used to estimate the spectral matrix, such as [6], [7].

Proposition V.2. Let $z_{k}$ be a non-derogatory ${ }^{9}$ eigenvalue of multiplicity $M_{k} \in \mathbb{N}^{*}$ of the $r \times r$ matrix $\boldsymbol{\Phi}(t)$, whose Jordan canonical form is $\boldsymbol{\Phi}(t)=\boldsymbol{G}(t) \boldsymbol{J} \boldsymbol{G}(t)^{-1}$. For all $\varepsilon>0$, let $\boldsymbol{\Phi}_{\varepsilon}(t)=\boldsymbol{\Phi}(t)+\varepsilon \Delta \boldsymbol{\Phi}(t)$, where $\Delta \boldsymbol{\Phi}(t)$ is an arbitrary $r \times r$ matrix. Then define the $r \times r$ matrix $\Delta \boldsymbol{J}=\boldsymbol{G}(t)^{-1} \Delta \boldsymbol{\Phi} \boldsymbol{G}(t)$. Let $\Delta J_{k}$ be the element of $\Delta \boldsymbol{J}$ which belongs to the row of index $\sum_{k^{\prime}=0}^{k-1} M_{k^{\prime}}$ and the column of index $\sum_{k^{\prime}=0}^{k} M_{k^{\prime}}-1$.

Suppose that $\Delta J_{k} \neq 0$. Then there exists a positive $\varepsilon_{0}$ such that for all $\varepsilon<\varepsilon_{0}$ there are exactly $M_{k}$ eigenvalues of $\boldsymbol{\Phi}_{\varepsilon}(t)$, denoted $\left\{z_{(k, m)}(\varepsilon)\right\}_{m \in\left\{0 \ldots M_{k}-1\right\}}$, which admit the first-order fractional expansion

$$
z_{(k, m)}(\varepsilon)=z_{k}+\varepsilon^{\frac{1}{M_{k}}} \Delta z_{k} e^{i 2 \pi \frac{m}{M_{k}}}+O\left(\varepsilon^{\frac{2}{M_{k}}}\right)
$$

where $\Delta z_{k}$ is an arbitrary $M_{k}^{\text {th }}$ root of $\Delta J_{k}$.

This proposition is a corollary of theorem 2.1 in [36], in the particular case of non-derogatory eigenvalues. Its proof can be found in [20]. If $M_{k}>1$, the first order perturbation of $z_{k}$ in equation (20) looks like that in equation (19) : it is homogeneous and isotropic, so that the $M_{k}$ perturbed eigenvalues form the vertices of a $M_{k}^{\text {th }}$ order regular polygon in the complex plane. As mentioned in section V-A, multiple poles appear to be more sensitive to perturbations than single poles, since the first order term in equation (20) is $\varepsilon^{\frac{1}{M_{k}}}$. In fact, this apparent sensitivity can be circumvented by computing the arithmetic mean of the estimated eigenvalues, as shown in proposition V.3.

Proposition V.3. Let $z_{k}(\varepsilon)=\frac{1}{M_{k}} \sum_{m=0}^{M_{k}} z_{(k, m)}(\varepsilon)$. Let $\Delta \boldsymbol{J}_{k}$ be the $M_{k} \times M_{k}$ matrix extracted from $\Delta \boldsymbol{J}$, which corresponds to the rows and columns of indices $\sum_{k^{\prime}=0}^{k-1} M_{k^{\prime}}$ to $\sum_{k^{\prime}=0}^{k} M_{k^{\prime}}-1$.

Suppose that $\operatorname{trace}\left(\Delta \boldsymbol{J}_{k}\right) \neq 0$. Then for all $\varepsilon<\varepsilon_{0}$, the function $\varepsilon \mapsto z_{k}(\varepsilon)$ admits the first order expansion

$$
z_{(k, m)}(\varepsilon)=z_{k}+\varepsilon \underline{\Delta} z_{k}+O\left(\varepsilon^{2}\right)
$$

where $\triangleq z_{k}=\frac{1}{M_{k}} \operatorname{trace}\left(\Delta \boldsymbol{J}_{k}\right)$.

The proof of proposition V.3 can be found in [20]. This proposition confirms that multiple poles are not more sensitive to perturbations than single poles. Moreover, multiple poles can be estimated by computing the arithmetic mean of the scattered eigenvalues. Thus the generalized ESPRIT algorithm presented in section IV-C can be simplified in the following way :

- apply the classical ESPRIT algorithm for estimating the eigenvalues of the spectral matrix,

\footnotetext{
${ }^{9}$ An eigenvalue is non-derogatory if and only if it appears in only one Jordan block (see e.g. [36] for more details). Since the complex poles are distinct, all the eigenvalues in the Jordan form (16) are non-derogatory.
}

- compute the arithmetic mean of the estimated eigenvalues associated to multiple poles.

Since the computational complexity of the first step is much higher than that of the second step, the overall complexity of this generalized ESPRIT algorithm is the same as that of the classical ESPRIT algorithm.

\section{Simulation RESUlts}

In this section, the ESPRIT method is applied to realvalued signals. The real-valued signal model is presented in section VI-A. Then section VI-B illustrates a case of polynomial amplitude modulation, and section VI-C illustrates a case of both amplitude and frequency modulation.

\section{A. Real valued signal model}

In this section the signal model introduced in section II-A is applied to the particular case of real-valued signals. Since the prediction polynomial has real-valued coefficients, its roots can be partitioned into real poles and complex conjugate pairs of poles of same multiplicity. Thus, by grouping poles whose polar angles have the same absolute value, equation (4) can be rewritten in the form

$$
s(t)=\sum_{p=0}^{P-1} a_{p}(t) \cos \left(2 \pi f_{p} t\right)+b_{p}(t) \sin \left(2 \pi f_{p} t\right)
$$

where $P \leq K$ is the number of distinct frequencies $f_{p} \in$ $\left[0, \frac{1}{2}\right]$, and $\forall p \in\{0, \ldots, P-1\}$, both $a_{p}(t)$ and $b_{p}(t)$ belong to a class of parametric functions. More precisely, a function $g(t)$ of this class has the form

$$
g(t)=\sum_{q=0}^{Q-1} P_{q}[t] \exp \left(\delta_{q} t\right)
$$

where $Q \in \mathbb{N}^{*}$ is the number of poles of the same polar angle, all the damping factors $\delta_{q} \in \mathbb{R}$ are distinct, and $\forall q \in\{0, \ldots, Q-1\}, P_{q}$ is a polynomial with real valued coefficients. Then equation (21) can be written in the form

$$
s(t)=\sum_{p=0}^{P-1} A_{p}(t) \cos \left(2 \pi f_{p} t+\varphi_{p}(t)\right)
$$

where the time-varying amplitude $A_{p}(t)$ and phase $\varphi_{p}(t)$ of the $p^{\text {th }}$ sinusoid satisfy the equations

$$
\left\{\begin{array}{l}
a_{p}(t)=A_{p}(t) \cos \left(\varphi_{p}(t)\right) \\
b_{p}(t)=-A_{p}(t) \sin \left(\varphi_{p}(t)\right)
\end{array}\right.
$$

whose solutions are $^{10}$

$$
\left\{\begin{array}{l}
A_{p}(t)=\sqrt{a_{p}(t)^{2}+b_{p}(t)^{2}} \\
\varphi_{p}(t)=-2 \arctan \left(\frac{b_{p}(t)}{A_{p}(t)+a_{p}(t)}\right) .
\end{array}\right.
$$

Note that the signal model in equation (22) looks like that of McAulay and Quatieri [37]. However, in [37] $A_{p}(t)$ and $\varphi_{p}(t)$ are non-parametric functions such that $A_{p}(t)$ and $\frac{d \varphi_{p}}{d t}$

\footnotetext{
${ }^{10}$ Note that $\arctan \left(\frac{-b_{p}(t)}{a_{p}(t)}\right)=\varphi_{p}(t)$ only if $\left.\varphi_{p}(t) \in\right]-\frac{\pi}{2}, \frac{\pi}{2}[$. Conversely, the proposed inversion formula is valid for all $\left.\varphi_{p}(t) \in\right]-\pi, \pi[$.
} 
have slow variations and $A_{p}(t)$ is positive, whereas in our model $A_{p}(t)$ and $\varphi_{p}(t)$ are parametric functions.

Note that the variations of the instantaneous frequency $f_{p}^{i}$ of the $p^{\text {th }}$ sinusoid can be calculated analytically, by differentiating (23):

$$
f_{p}^{i}(t)=f_{p}+\frac{1}{2 \pi} \frac{d \varphi_{p}}{d t}=f_{p}+\frac{1}{2 \pi} \frac{\frac{d a_{p}}{d t} b_{p}(t)-\frac{d b_{p}}{d t} a_{p}(t)}{a_{p}(t)^{2}+b_{p}(t)^{2}} .
$$

Thus the PACE signal model consists of both amplitude and frequency modulated sinusoids. Equations (24) and (25) show that these amplitude and frequency modulations are closely related.

\section{B. Polynomial amplitude modulation}
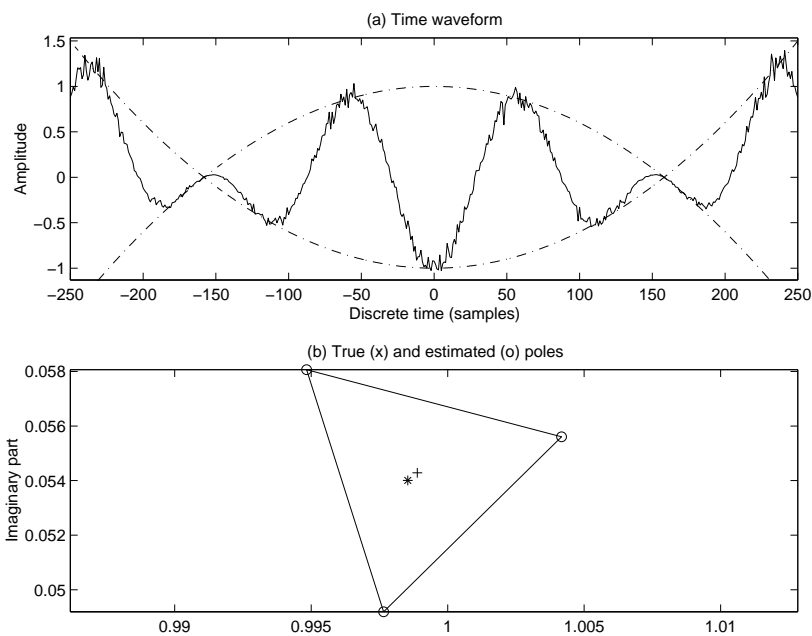

Fig. 1. Polynomial amplitude modulation

(a) Test signal (solid line) and its envelope (dashed line)

(b) True pole ( $\star$ ), scattered eigenvalues (o) and their mean $(+)$

The test signal shown in figure 1-a is a noisy single sinusoid with polynomial amplitude modulation and no frequency modulation (the dotted lines represent its envelope). More precisely, this signal is that defined in equation (21) with $P=1, f_{0}=8.6 \times 10^{-3}, a_{0}(t)=\frac{t^{2}}{25000}-1$ and $b_{0}(t)=0$, plus an additive white noise whose variance was chosen so that the Signal Noise Ratio (SNR) is $20 \mathrm{~dB}$. The corresponding complex model parameters are $K=2$ and $M_{0}=M_{1}=3$ (thus $r=6$ ), and the observation window is $t \in[-250,250]$.

The ESPRIT algorithm was applied with parameters $n=$ $l=251$. The three estimated eigenvalues with positive angles are represented in figure 1-b, by means of ' $O$ ' marks at the vertices of the triangle. The true multiple pole $z_{0}=e^{i 2 \pi f_{0}}$ is represented by a '*' mark. As mentioned in section $\mathrm{V}$ $\mathrm{B}$, it can be noticed that the first order perturbation of $z_{0}$ is approximately homogeneous and isotropic, so that $z_{0}$ is close to the arithmetic mean of the three estimated eigenvalues (represented by a + mark). The relative frequency deviation between the true pole and the arithmetic mean of the estimated eigenvalues is $0.48 \%$.
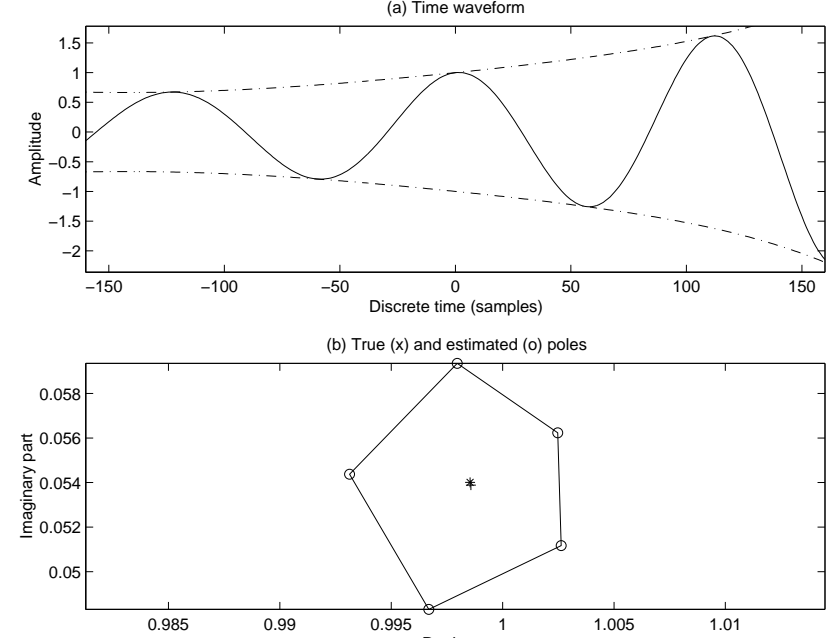

Fig. 2. Both amplitude and frequency moal part

(a) Test signal (solid line) and its envelope (dashed line)

(b) True pole $(\star)$, scattered eigenvalues (o) and their mean $(+)$

\section{Both amplitude and frequency modulation}

The test signal shown in figure 2 -a is that defined in equation (21) with $P=1, f_{0}=8.6 \times 10^{-3}$, and

$$
\left\{\begin{array}{l}
a_{0}(t) \triangleq\left(1+\delta_{0} t+\frac{1}{2} \delta_{0}^{2} t^{2}\right) \\
b_{0}(t) \triangleq-\pi \Delta f t^{2} a_{0}(t)
\end{array}\right.
$$

where $\delta_{0}=4 \times 10^{-3}, \Delta f=8 \times 10^{-6}$, plus an additive white noise whose variance was chosen so that the SNR is $50 \mathrm{~dB}^{11}$. The corresponding complex model parameters are $K=2$ and $M_{0}=M_{1}=5$ (thus $r=10$ ), and the observation window is $t \in[-500,500]^{12}$.

Equations (24) and (25) yield the corresponding amplitude and frequency modulations:

$$
\left\{\begin{aligned}
A_{0}(t) & =\sqrt{1+\pi^{2} \Delta f^{2} t^{4}} a_{0}(t) \\
f_{0}^{i}(t) & =f_{0}+\frac{\Delta f t}{1+\pi^{2} \Delta f^{2} t^{4}} .
\end{aligned}\right.
$$

In particular, the observation window of figure 2-a shows both an amplitude and frequency increase.

The ESPRIT method was applied with parameters $n=$ $l=501$. The five estimated eigenvalues with positive angles are represented in figure 2-b, by means of ' $O$ ' marks at the vertices of the pentagon. The true multiple pole $z_{0}=e^{i 2 \pi f_{0}}$ is represented by a '*' mark. As mentioned in section $\mathrm{V}$ $\mathrm{B}$, it can be noticed that the first order perturbation of $z_{0}$ is approximately homogeneous and isotropic, so that $z_{0}$ is close to the arithmetic mean of the five estimated eigenvalues. In fact, the relative frequency deviation between the true pole and the arithmetic mean of the estimated eigenvalues is $0.23 \%$.

\section{CONCLUSIONS}

In this paper we introduced the Polynomial Amplitude Complex Exponentials (PACE) signal model as the general

\footnotetext{
${ }^{11}$ Since the multiplicity of the poles is higher than in section VI-B, proposition V.2 shows that the scattering of the eigenvalues is emphasized. Thus we chose a higher SNR to obtain a similar result (a SNR of $20 \mathrm{~dB}$ is not sufficient to obtain an homogeneous and isotropic scattering).

${ }^{12}$ Figure 2-a zooms in on the central part of the signal.
} 
solution to homogeneous linear recursions. This model extends the well known Exponential Sinusoidal Model (ESM) to the case of multiple poles, and represents the signal as a sum of both amplitude and frequency modulated sinusoids. A general factorization of Hankel matrices related to this model was proposed, which involves Pascal-Vandermonde matrices. Based on the rotational invariance property of such matrices, a generalized ESPRIT algorithm for estimating the signal poles was proposed, involving the Jordan canonical form of the spectral matrix.

In presence of noise, the multiple poles are scattered into several single eigenvalues, forming the vertices of a regular polygon as a first order approximation. This phenomenon was observed in our numerical simulations, which confirmed that the arithmetic mean of the scattered eigenvalues is a good approximation of the original multiple pole. Therefore the PACE model leads to an alternative interpretation of a set of estimated eigenvalues belonging to the same neighborhood (several single eigenvalues can correspond to a single modulated sinusoid).

In other respects, it can be noticed that the specific amplitude and frequency modulations involved in the PACE model are closely related. This might suggest that this model is not appropriate for independent phase and envelope modulations. In practice, we observed that single poles were generally sufficient for representing chirps and sinusoidal modulations (like tremolo and vibrato in music signals). However, it is well known that complex polynomials can uniformly approximate any continuous complex function in a closed and bounded interval $^{13}$. Thus the PACE model might be appropriate for coding arbitrary frequency and amplitude modulations on short time windows. Indeed, we found some audio signals (e.g. violin vibratos and guitar attacks) which could be coded more efficiently with multiples poles than single poles (i.e. more precisely or with less parameters) on very short windows $(5$ $\mathrm{ms}$ ), but most often the best results are obtained with single poles only. As a conclusion, the PACE model offers interesting outlooks for signal processing, but its application to audio coding is not straightforward.

\section{APPENDIX}

\section{A. Factorization of the data matrix}

The following lemma, known as the binomial identity [18], [19], will be involved in the proof of proposition III.7 below. It can be proved by induction over $m$ [20].

Lemma A.1 (Binomial identity). For all $m \in \mathbb{N}$, the falling factorials satisfy the identity

$$
F_{m}[X+Y]=\sum_{m^{\prime}=0}^{m} F_{m^{\prime}}[X] F_{m-m^{\prime}}[Y] .
$$

Proof of proposition III.7. The coefficients of the matrix $\boldsymbol{S}(t)$ are $\forall i \in\{0 \ldots n-1\}, \forall j \in\{0 \ldots l-1\}$

$$
\boldsymbol{S}(t)_{(i, j)}=s(t-l+1+i+j)
$$

\footnotetext{
${ }^{13}$ This result is known as the Weierstrass approximation theorem.
}

Substituting equation (6) into equation (26) yields

$$
\boldsymbol{S}(t)=\sum_{k=0}^{K-1} \boldsymbol{S}_{k}(t)
$$

where the coefficients of the $n \times l$ matrix $\boldsymbol{S}_{k}(t)$ are

$$
\boldsymbol{S}_{k}(t)_{(i, j)}=\sum_{m=0}^{M_{k}-1} \alpha_{(k, m)} F_{m}[t-l+1+i+j] z_{k}{ }^{t-l+1+i+j-m}
$$

Then lemma A.1 yields

$F_{m}[t-l+1+i+j]=\sum_{m^{\prime}=0}^{m} F_{m^{\prime}}[i+j] F_{m-m^{\prime}}[t-l+1]$

Substituting equations (29) and (10) into equation (28) shows that

$$
\boldsymbol{S}_{k}(t)_{(i, j)}=\sum_{m^{\prime}=0}^{M_{k}-1} \beta_{k, m^{\prime}}(t) F_{m^{\prime}}[i+j] z_{k}^{i+j-m^{\prime}} .
$$

Applying lemma A.1 again yields

$$
F_{m^{\prime}}[i+j]=\sum_{m^{\prime \prime}=0}^{m^{\prime}} F_{m^{\prime \prime}}[i] F_{m^{\prime}-m^{\prime \prime}}[j]
$$

Then substituting equation (31) into equation (30) yields

$$
\begin{aligned}
& \boldsymbol{S}_{k}(t)_{(i, j)}= \\
& \sum_{m^{\prime}=0}^{M_{k}-1} \beta_{k, m^{\prime}}(t) \sum_{m^{\prime \prime}=0}^{m^{\prime}} \boldsymbol{C}_{M_{k}}^{n}\left(z_{k}\right)_{\left(i, m^{\prime \prime}\right)} \boldsymbol{C}_{M_{k}}^{l}\left(z_{k}\right)_{\left(j, m^{\prime}-m^{\prime \prime}\right)}
\end{aligned}
$$

which can be written as a product of matrices:

$$
\boldsymbol{S}_{k}(t)=\boldsymbol{C}_{M_{k}}^{n}\left(z_{k}\right) \boldsymbol{H}_{k}(t) \boldsymbol{C}_{M_{k}}^{l}\left(z_{k}\right)^{T} .
$$

Substituting equation (32) into equation (27) finally yields factorization (8).

Proof of corollary III.8. Proposition III.6 shows that both $\boldsymbol{V}^{n}$ and $\boldsymbol{V}^{l}$ have rank $r$. Consequently, factorization (8) shows that $\boldsymbol{S}(t)$ has rank $r$ if and only if the $r \times r$ matrix $\boldsymbol{D}(t)$ is nonsingular. Besides, equation (9) shows that $\boldsymbol{D}(t)$ is non-singular if and only if $\boldsymbol{H}_{k}(t)$ is non singular $\forall k \in\{0 \ldots K-1\}$. Since $\boldsymbol{H}_{k}(t)$ is upper anti-triangular with all anti-diagonal coefficients equal to $\beta_{\left(k, M_{k}-1\right)}, \boldsymbol{H}_{k}(t)$ is non-singular if and only if $\beta_{\left(k, M_{k}-1\right)} \neq 0$. Moreover, equation (10) shows that $\forall k \in\{0 \ldots K-1\}, \beta_{\left(k, M_{k}-1\right)}=\alpha_{\left(k, M_{k}-1\right)} z_{k}^{t-(l-1)}$. It follows that $\boldsymbol{D}(t)$ is non-singular if and only if $\alpha_{\left(k, M_{k}-1\right)} \neq 0$ $\forall k \in\{0 \ldots K-1\}$.

\section{B. Rotational Invariance Property of generalized Pascal ma- trices}

The following lemma is used to show the rotational invariance property of Pascal-Vandermonde matrices in section IV.

Lemma B.1 (Rotational Invariance Property of generalized Pascal matrices). Suppose that $n \geq 2$. Let $C_{M}^{n}(z)_{\downarrow}$ be the matrix extracted from $C_{M}^{n}(z)$ by deleting the last row. Similarly, let $C_{M}^{n}(z)_{\uparrow}$ be the matrix extracted from $C_{M}^{n}(z)$ 
by deleting the first row. Then $C_{M}^{n}(z)_{\downarrow}$ and $C_{M}^{n}(z) \uparrow$ span the same subspace, and

$$
C_{M}^{n}(z)_{\uparrow}=C_{M}^{n}(z)_{\downarrow} \boldsymbol{J}_{M}(z) .
$$

Proof. The coefficients of the matrix $C_{M}^{n}(z) \uparrow$ are defined as $C_{M}^{n}(z)_{\uparrow(i, j)}=F_{j}[i+1] z^{(i+1)-j}$. Moreover, equation (5) shows that $F_{j}[i+1]=F_{j}[i]+F_{(j-1)}[i]$. Consequently,

$$
\begin{aligned}
C_{M}^{n}(z)_{\uparrow(i, j)} & =z F_{j}[i] z^{i-j}+F_{j-1}[i] z^{i-(j-1)} \\
& =z C_{M}^{n}(z)_{\downarrow(i, j)}+\mathbf{1}_{\{j \geq 1\}} C_{M}^{n}(z)_{\downarrow(i, j-1)}
\end{aligned}
$$

This last equation can be written in the form (33).

\section{REFERENCES}

[1] G. M. Riche de Prony, "Essai expérimental et analytique: sur les lois de la dilatabilité de fluides élastiques et sur celles de la force expansive de la vapeur de l'eau et de la vapeur de l'alcool à différentes températures," Journal de l'école polytechnique, vol. 1, no. 22, pp. 24-76, 1795, in French.

[2] V. F. Pisarenko, "The retrieval of harmonics from a covariance function," Geophysical J. Royal Astron. Soc., vol. 33, pp. 347-366, 1973.

[3] R. O. Schmidt, "Multiple emitter location and signal parameter estimation," IEEE Trans. Antennas Propagat., vol. 34, no. 3, pp. 276-280, Mar. 1986.

[4] A. J. Barabell, "Improving the resolution performance of eigenstructurebased direction-finding algorithms," in Proc. of ICASSP'83. Boston, MA: IEEE, 1983, pp. 336-339.

[5] S. Y. Kung, K. S. Arun, and D. B. Rao, "State-space and singular value decomposition based approximation methods for harmonic retrieval problem," J. of Opt. Soc. of America, vol. 73, pp. 1799-1811, Dec. 1983.

[6] R. Roy, A. Paulraj, and T. Kailath, "ESPRIT-A subspace rotation approach to estimation of parameters of cisoids in noise," IEEE Trans. Acoust., Speech, Signal Processing, vol. 34, no. 5, pp. 1340-1342, Oct. 1986.

[7] R. Roy and T. Kailath, "Total least squares ESPRIT," in Proc. of 21 st Asilomar Conference on Signals, Systems, and Computers, Nov. 1987, pp. 297-301.

[8] M. Zoltawski and D. Stavrinides, "Sensor array signal processing via a Procrustes rotations based eigen-analysis of the ESPRIT data pencil," IEEE Trans. Acoust., Speech, Signal Processing, vol. 37, no. 6, pp. 832861, June 1989.

[9] J. Nieuwenhuijse, R. Heusens, and E. F. Deprettere, "Robust exponential modeling of audio signals," in Proc. of ICASSP'98, vol. 6. IEEE, May 1998, pp. 3581-3584.

[10] J. Jensen, R. Heusdens, and S. H. Jensen, "A perceptual subspace approach for modeling of speech and audio signals with damped sinusoids," IEEE Trans. Speech Audio Processing, vol. 12, no. 2, pp. 121-132, Mar. 2004.

[11] K. Hermus, W. Verhelst, P. Lemmerling, P. Wambacq, and S. Van Huffel, "Perceptual audio modeling with exponentially damped sinusoids," Signal processing, vol. 85, no. 1, pp. 163-176, Jan. 2005.

[12] R. Kumaresan and D. W. Tufts, "Estimating the parameters of exponentially damped sinusoids and pole-zero modeling in noise," IEEE Trans. Acoust., Speech, Signal Processing, vol. 30, no. 6, pp. 833-840, Dec. 1982.

[13] Y. Hua and T. K. Sarkar, "Matrix pencil method for estimating parameters of exponentially damped/undamped sinusoids in noise," IEEE Trans. Acoust., Speech, Signal Processing, vol. 38, no. 5, pp. 814-824, May 1990.

[14] Y. Li, K. Liu, and J. Razavilar, "A parameter estimation scheme for damped sinusoidal signals based on low-rank Hankel approximation," IEEE Trans. Signal Processing, vol. 45, pp. 481-486, Feb. 1997.

[15] A.-J. Van der Veen, E. F. Deprettere, and A. L. Swindlehurst, "Subspace based signal analysis using singular value decomposition," Proc. of IEEE, vol. 81, no. 9, pp. 1277-1308, Sept. 1993.

[16] M. Goodwin, "Matching pursuit with damped sinusoids," in Proc. of ICASSP'97, Munich, Germany, Apr. 1997, pp. 2037-2040, vol. 3.
[17] D. Kincaid and W. Cheney, Numerical analysis, 2nd ed. Pacific Grove, California: Brooks/Cole Publishing, 1996.

[18] S. Roman, The Umbral Calculus. New York: Academic Press, 1984, §1.2: The Lower Factorial Polynomial.

[19] R. L. Graham, D. E. Knuth, and O. Patashnik, Concrete Mathematics: A Foundation for Computer Science, 2nd ed. Reading, MA: AddisonWesley, 1994.

[20] R. Badeau, "High Resolution methods for estimating and tracking modulated sinusoids. Application to music signals." Ph.D. dissertation, École Nationale Supérieure des Télécommunications, Paris, Apr. 2005, in french.

[21] G. Strang, Introduction to Linear Algebra, 3rd ed. Wellesley-Cambridge Press, 2003.

[22] R. A. Horn and C. R. Johnson, Matrix analysis. Cambridge University Press, 1985.

[23] D. L. Boley, F. T. Luk, and D. Vandevoorde, "A General Vandermonde Factorization of a Hankel Matrix," in Int'l Lin. Alg. Soc. (ILAS) Symp. on Fast Algorithms for Control, Signals and Image Processing, Winnipeg, Canada, 1997.

[24] D. Vandevoorde, "A fast exponential decomposition algorithm and its applications to structured matrices," Ph.D. dissertation, Rensselaer Polytechnic Institute, Troy, New York, 1996.

[25] R. Badeau, G. Richard, and B. David, "Sliding window adaptive SVD algorithms," IEEE Trans. Signal Processing, vol. 52, no. 1, pp. 1-10, Jan. 2004.

[26] R. Badeau, B. David, and G. Richard, "Yet Another Subspace Tracker," in Proc. of ICASSP'05, vol. 4. IEEE, Mar. 2005, pp. 329-332.

[27] — - "Fast Approximated Power Iteration Subspace Tracking," IEEE Trans. Signal Processing, vol. 53, no. 8, Aug. 2005.

[28] G. Bienvenu and L. Kopp, "Optimality of high-resolution array processing using the eigensystem method," IEEE Trans. Acoust., Speech, Signal Processing, vol. 31, no. 5, pp. 1235-1245, Oct. 1983.

[29] M. Wax and T. Kailath, "Detection of signals by information theoretic criteria," IEEE Trans. Acoust., Speech, Signal Processing, vol. 33, no. 2, pp. 387-392, Apr. 1985.

[30] L. C. Zhao, P. R. Krishnaiah, and Z. D. Bai, "On detection of the number of signals in presence of white noise," Journal of Multivariate Analysis, vol. 20, no. 1, pp. 1-25, 1986.

[31] R. Badeau, B. David, and G. Richard, "Selecting the modeling order for the ESPRIT high resolution method: an alternative approach," in Proc. of ICASSP'04, vol. 2. Montreal, Quebec: IEEE, May 2004, pp. $1025-1028$.

[32] _ " "A new perturbation analysis for signal enumeration in rotational invariance techniques," IEEE Trans. Signal Processing, 2005, to be published.

[33] P. Stoica, H. Li, and J. li, "Amplitude estimation of sinusoidal signals: survey, new results, and an application," IEEE Trans. Signal Processing, vol. 48, no. 2, pp. 338-352, 2000.

[34] J. H. Wilkinson, The Algebraic Eigenvalue Problem. Oxford, UK: Clarendon Press, 1988.

[35] Z. Zeng, "Computing multiple roots of inexact polynomials," in ISSAC'03. Philadelphia, Pennsylvania, USA: ACM, Aug. 2003.

[36] J. Moro, J. V. Burke, and M. L. Overton, "On the Lidskii-VishikLyusternik perturbation theory for eigenvalues of matrices with arbitrary Jordan structure," SIAM Journal on Matrix Analysis and Applications, vol. 18, no. 4, pp. 793-817, Oct. 1997.

[37] R. J. Mc Aulay and T. F. Quatieri, "Speech analysis and synthesis based on a sinusoidal representation," IEEE Trans. Acoust., Speech, Signal Processing, vol. 34, no. 4, pp. 744-754, Aug. 1986. 